\title{
Atendimento à saúde de crianças e adolescentes em situaçãa de violência sexual, em quatro capitais brasileiras
}

Suely Ferreira Deslandes(a)

Luiza Jane Eyre de Souza Vieira(b)

Ludmila Fontenele Cavalcanti ${ }^{(c)}$

Raimunda Magalhães da Silva(d)

Deslandes SF, Vieira LJES, Cavalcanti LF, Silva RM. Health care of children and adolescents in situation of sexual violence, in four Brazilian capitals. Interface (Botucatu). 2016;

20(59):865-77.

The paper analyzes the health care of children and adolescents in situation of sexual violence (SV) in the municipal public health network of four Brazilian cities - Porto Alegre (RS), Belém (PA), Fortaleza (CE) and Campo Grande (MS) -, focusing on the availability of services and the provision of care, such as hormonal emergency contraception (HEC), prophylaxis for sexually transmitted diseases (STDs) and legal termination of pregnancy (LTP). The case study method chosen was of semi-structured interviews, with twenty managers and technicians from the central level, representatives of the municipal health secretariats, as well as document analysis. The provision of care is uneven across the cities. Considering the high demand, there is low supply of services in three of them. The specialized care, as HEC and legal termination of pregnancy (LTP) still face resistance of professionals and religious sectors. The article highlights innovations of two Integrated Centers of Attention and a Psychosocial Care Center Post Trauma.

Keywords: Health Care. Child and adolescent. Sexual violence. Health Services.
O artigo analisa a atenção à saúde de crianças e adolescentes em situação de violência sexual (VS) na rede pública municipal de saúde de quatro capitais brasileiras - Porto Alegre (RS), Belém (PA), Fortaleza (CE) e Campo Grande (MS) -, enfocando a disponibilidade de serviços e a oferta de atendimentos, tais como a anticoncepção de emergência hormonal $(\mathrm{AEH})$, profilaxias para doenças sexualmente transmissíveis (DSTs) e interrupção legal da gravidez (ILG). O método de estudo de casos foi conduzido por entrevistas semiestruturadas, com vinte gestores e técnicos do nível central, representantes das secretarias municipais de saúde, e de análise documental. A atenção é desigual entre as capitais. Considerando-se a alta demanda, há baixa oferta de serviços em três delas. Os atendimentos especializados, como AEH e ILG, encontram resistências dos profissionais e setores religiosos. Destacamse inovações de dois Centros Integrados de Atenção e de um Centro de Atenção Psicossocial Pós-Trauma.

Palavras-chave: Atenção à Saúde. Criança e adolescente. Violência sexual. Serviços de Saúde. (a) Instituto Fernandes Figueira, Fundação Oswaldo Cruz. Av Rui Barbosa, 716/2 andar - Departamento de Ensino, Flamengo. Rio de Janeiro, RJ, Brasil. 22250-020. deslandes.s@gmail.com (b,d) Programa de PósGraduação em Saúde Coletiva, Universidade de Fortaleza. Fortaleza, CE, Brasil. janeeyre@ unifor.br; rmsilva@ unifor.br

(c) Programa de PósGraduação em Serviço Social, Escola de Serviço Social, Universidade Federal do Rio de Janeiro. Rio de Janeiro, RJ, Brasil. ludmila.ufrj@ gmail.com 


\section{Introdução}

A violência sexual (VS) é definida pelas agências brasileiras e internacionais de saúde como todo ato ou jogo sexual de cujo agressor se encontra em estágio de desenvolvimento psicossexual mais adiantado do que o da criança ou adolescente envolvido. Tem como intenção estimulá-lo sexualmente ou utilizá-lo para obter satisfação sexual. Baseia-se em desiguais relações de poder (de força, inteligência, autoridade), podendo se manifestar de diversas formas (com ou sem contato físico, com ou sem conjunção carnal) $)^{1,2}$.

Os danos físicos e emocionais da VS a crianças e adolescentes variam: desde doenças sexualmente transmissíveis (DSTs), lesões geniturinárias e gravidezes indesejadas até manifestações como desconfiança, dificuldade de expressão, baixa autoestima, isolamento, comportamento agressivo e/ou defensivo, retraimento e depressão ${ }^{3,4}$.

A VS é reconhecida como uma violação dos direitos humanos e dos direitos sexuais, que impede crianças e adolescentes de usufruírem de uma sexualidade compatível com seu estágio de desenvolvimento, livre de discriminação ou coerção. Viola o direito reprodutivo de adolescentes de decidirem livre e responsavelmente sobre terem filhos (quantos e quando), bem como o acesso à informação e aos meios para a tomada desta decisão ${ }^{5,6}$.

A incidência do fenômeno VS em crianças e adolescentes aponta o risco maior de vitimização de meninas, evidenciando assimetrias de gênero ${ }^{7}$, incorporando representações sociais historicamente construídas e relações de dominação, que designam a homens e mulheres papéis e oportunidades desiguais na sociedade.

Segundo estimativa do UNICEF, em 2014, no contexto mundial, cerca de cento e vinte milhões de meninas com idades até 19 anos foram submetidas à VS pelo menos uma vez na vida. Inexiste estimativa global sobre meninos, apesar de também estarem expostos a este tipo de violência8.

No Brasil, no biênio 2011-2012, registraram-se 112.394 denúncias de VS contra criança e adolescente, pelo serviço nacional de denúncia, o Disque $100^{9}$.

O setor Saúde constitui, entre as políticas e serviços públicos, o locus para onde converge a maior parte das vítimas de violências ${ }^{10}$. Cerca de vinte casos de crianças até nove anos, vítimas de VS, são notificados diariamente na rede pública de saúde. Registros do Sistema de Informação de Agravos de Notificação (SINAN) mostram que, em 2012, houve 7.592 notificações de VS nessa faixa etária, correspondendo a $27 \%$ dos casos de violência infantil registrados. Nesse mesmo ano, na faixa etária de dez a 19 anos, foram notificados 9.919 casos de abuso sexual ${ }^{11}$. Todavia, muitas situações de VS contra esses grupos não chegam à rede pública de saúde ou não são identificadas pelos profissionais.

A organização da atenção integral à saúde de crianças e adolescentes em situação de VS requer uma dinâmica complexa, pois demanda o atendimento a questões clínicas, psicológicas, sociais e legais, visando a continuidade do atendimento e restauração de direitos violados. Requer, ainda, a articulação das ações desenvolvidas pela saúde com as de proteção social e com as demais agências e atores do Sistema de Garantia de Direitos da Criança e do Adolescente nos territórios ${ }^{1,12,13}$.

O próprio Ministério da Saúde reconhece que, por ser um dos espaços privilegiados para a identificação de mulheres, adolescentes e crianças em situação de VS, o setor tem papel fundamental na definição e na articulação dos serviços e organizações, que, direta ou indiretamente, atendem essas situações ${ }^{14}$. A organização desses serviços é de responsabilidade do gestor local da saúde, cuja atuação é normatizada pela Norma Técnica de Prevenção e Tratamento dos Agravos Resultantes da Violência Sexual contra Mulheres e Adolescentes (NT) ${ }^{14}$. A NT regula os atendimentos clínico e psicossocial, que devem ser prestados a partir de uma postura de acolhimento, escuta ativa e discrição. A NT também orienta sobre as instalações físicas, os recursos humanos e os medicamentos a serem disponibilizados na unidade de saúde às vítimas de VS, visando a profilaxia das DSTs e dos demais agravos delas decorrentes.

Percebe-se o aumento da produção científica nacional sobre VS contra crianças e adolescentes. Entretanto, ainda são incipientes os estudos sobre a atenção à saúde desse segmento, na perspectiva da atenção nos serviços de saúde. Levantamento realizado em janeiro de 2015, na base Scielo, 
tomando a VS como assunto e incluindo o descritor "atenção", resultou em 13 artigos. Porém, quando refinada a busca para adolescentes, apenas três deles permaneceram.

Nesse sentido, este artigo analisa a atenção à saúde de crianças e adolescentes em situação de VS na rede pública municipal de saúde de quatro capitais brasileiras, enfocando a composição e a articulação entre serviços e a oferta de atendimentos estratégicos, entre 2010-12, tais como: a anticoncepção de emergência hormonal (AEH), profilaxias para DSTs e interrupção legal da gravidez (ILG).

\section{Método}

O presente artigo insere-se na pesquisa multicêntrica denominada "Avaliação das estratégias governamentais municipais no enfrentamento da exploração sexual de crianças e adolescentes em quatro capitais das macrorregiões brasileiras". Este estudo focou os cinco eixos da atuação dos governos municipais no enfrentamento às violências (1 Prevenção e promoção de vínculos cuidadores; 2 Atenção às crianças e aos adolescentes em situação de violência; 3 Fortalecimento do sistema de garantia de direitos; 4 Registro e notificação; e 5 Responsabilização dos autores). Utilizou um conjunto de indicadores construídos com o apoio de especialistas e por meio de metodologias participativas ${ }^{15}$, aplicados em pesquisas anteriores e avaliados quanto ao seu desempenho ${ }^{16}$. Este artigo enfoca unicamente o eixo de atendimento às crianças e aos adolescentes em situação de VS.

O estudo de caso ${ }^{17}$ adotado constitui método investigativo comumente empregado pelas ciências sociais. Esse método possibilita um aprofundamento tanto exploratório quanto analítico acerca de um objeto delimitado geográfica e temporalmente. Alia, ao percurso interpretativo, teorias, práticas ou experiências adquiridas pelos pesquisadores; permite aprofundamento na investigação empírica; apresenta procedimentos metodológicos flexíveis; e pode utilizar técnicas da pesquisa histórica, da análise de documentos, de observações e de entrevistas 17,18 .

O estudo é composto por quatro casos selecionados pelos critérios de diversidade regional, alta incidência de denúncias de VS e exploração sexual, e aceitação das prefeituras em participar da pesquisa. Para a seleção das capitais, foram consultadas informações oriundas do serviço nacional de denúncias Disque 100, considerando-se a soma de denúncias no período de 2003 a 2010. Assim, selecionaramse as capitais Porto Alegre (RS), Campo Grande (MS), Belém (PA) e Fortaleza (CE), garantindo a representação de quatro das cinco regiões brasileiras. A Região Sudeste não foi incluída por ter sido analisada em estudo anterior, atuando como teste dos indicadores aplicados. A análise considerou a existência de serviços da rede pública municipal de saúde para atenção às crianças e aos adolescentes em situação de VS e a oferta de atendimentos clínicos e psicossociais dispensada a esse grupo.

A pesquisa foi apresentada em seminário a todas as secretarias de governo com atuação na área da infância. A entrada em campo se deu somente após a assinatura do termo de adesão de cada prefeitura.

A coleta de dados, realizada no período de junho a novembro de 2012, pautou-se na aplicação de entrevistas e análise de documentos produzidos pelas secretarias. A coleta foi feita por equipes locais, lideradas por pesquisador sênior com formação em Saúde Coletiva.

Foram realizadas vinte entrevistas com gestores e técnicos do nível central das secretarias municipais de saúde. O critério de inclusão do entrevistado foi o de ser gestor da secretaria de saúde (secretário ou subsecretário) ou representante oficialmente designado. Também foram selecionados os coordenadores de serviços de referência ao atendimento às crianças e aos adolescentes em situação de VS. Não houve recusa ao estudo.

As entrevistas ocorreram em duas sessões, com intervalo médio de dois meses e com duração média de 35 minutos. O roteiro da primeira entrevista foi semiestruturado e tratou dos seguintes temas: oferta e disponibilidades de serviços, atuação intersetorial para o atendimento, oferta de profilaxias para DST/Aids, interrupção da gravidez e AEH. O segundo roteiro foi aberto incluindo questões de esclarecimento e confirmação sobre as informações da primeira entrevista. As entrevistas foram transcritas e tomadas como fontes documentais orais. 
Além dos depoimentos, os documentos das secretarias de saúde que caracterizassem a rede de atenção e a oferta de serviços relatados pelos gestores foram categorizados segundo preceitos da análise temática ${ }^{19}$.

A categorização do acervo foi orientada pela base normativa contida nas políticas, planos e notas técnicas que preconizam a atenção às pessoas em situação de VS, com ênfase nas orientações da NT $T^{14}$. O período considerado no estudo foi o de 2010 a 2012. Assim, para a análise, foram definidas duas unidades temáticas, com seus subtemas: (1) caracterização da rede municipal de saúde na atenção às crianças e aos adolescentes em situação de VS, abrangendo (a) unidades do Sistema Único de Saúde que façam tal atendimento e (b) a existência de um fluxo intersetorial de atenção e a participação do setor Saúde; (2) oferta de atendimento às crianças e aos adolescentes em situação de VS, incluindo (a) $\mathrm{AEH}$, (b) ILG e (c) oferta e fluxo para profilaxias DST/Aids e hepatites virais.

Os resultados foram dispostos descritivamente, em um primeiro nível, agrupados pelas temáticas e subtemas identificados, destacando, individualmente, cada capital. Posteriormente, as principais tendências e questões observadas foram confrontadas com a literatura. Todos os resultados foram apresentados em sessão pública aos gestores e técnicos participantes do estudo, a fim de confirmar as informações e identificar eventuais correções necessárias.

O projeto obteve sua aprovação pelo Comitê de Ética e Pesquisa do Instituto Fernandes Figueira, da Fundação Oswaldo Cruz.

\section{Resultados}

Cada uma das quatro capitais estudadas apresentou, em 201020, Índice de Desenvolvimento Humano Municipal considerado alto, variando de 0,729, em Belém, a 0,805, em Porto Alegre. Embora o Índice de Gini da renda domiciliar per capita registrado em cada capital aponte uma variação positiva na última década, foram mantidos patamares de alta desigualdade econômica, de 0,56, em Campo Grande, a 0,61, em Porto Alegre e Fortaleza. Este cenário é afetado pela desigual disponibilidade de serviços públicos nos territórios. A variação do percentual de mães chefes de família sem Ensino Fundamental e com filho menor, no total de mães nessa categoria, variou de 11,70, em Campo Grande, a 19,39, em Fortaleza. Tais características sinalizam cenários de vulnerabilidade social para o segmento infantojuvenil.

\section{Breve caracterização da demanda e da oferta na rede de saúde para a atenção às crianças e aos adolescentes em situação de VS}

Considerando-se as notificações de VS realizadas pelos serviços de saúde (Tabela 1) no período estudado, verifica-se elevado número de casos, especialmente em Belém e Porto Alegre. Em contrapartida, Fortaleza, provavelmente devido à baixa implantação da "Ficha de notificação/ investigação individual de violência doméstica, sexual e/ou outras violências", apresentou poucos casos.

Os gestores afirmaram que a maioria dos serviços da rede básica municipal oferecia algum atendimento a crianças e adolescentes em situação de VS. Todavia, os serviços de referência apresentaram diferenças entre as capitais, com destaque para Campo Grande e Porto Alegre, pelo maior número de unidades apresentado (Quadro 1).

Belém, com menor número de unidades de referência, informou que os serviços da rede municipal apenas realizavam o acolhimento básico e a notificação. As unidades encaminhavam para a realização das profilaxias no hospital da Santa Casa de Misericórdia do Pará (FSCMP), gerenciada pela rede estadual. Lá funciona o Programa PROPAZ, especializado no atendimento à VS, que constitui um centro integrado de atendimento e inclui um posto do Instituto Médico Legal (IML). Quando se passavam 72 horas da ocorrência, o caso era enviado para a Rede Municipal de Atenção para Infecções Sexualmente Transmissíveis, Aids e hepatites virais, em um único serviço, a Casa Dia. 
Tabela 1. Distribuição dos casos de violência sexual por sexo, segundo município de notificação, Belém, Campo Grande, Fortaleza e Porto Alegre, 2010-2012.

\begin{tabular}{|c|c|c|c|c|c|c|c|c|}
\hline \multirow[b]{2}{*}{ Características } & \multicolumn{2}{|c|}{ Belém } & \multicolumn{2}{|c|}{ Campo Grande } & \multicolumn{2}{|c|}{ Fortaleza } & \multicolumn{2}{|c|}{ Porto Alegre } \\
\hline & $\begin{array}{c}\text { Masculino } \\
\mathrm{N}\end{array}$ & $\underset{\mathrm{N}}{\text { Feminino }}$ & $\begin{array}{c}\text { Masculino } \\
\mathrm{N}\end{array}$ & $\underset{\mathrm{N}}{\text { Feminino }}$ & $\begin{array}{c}\text { Masculino } \\
\mathrm{N}\end{array}$ & $\underset{N}{\text { Feminino }}$ & $\begin{array}{c}\text { Masculino } \\
\mathrm{N}\end{array}$ & $\underset{\mathrm{N}}{\text { Feminino }}$ \\
\hline \multicolumn{9}{|l|}{ Faixa Etária } \\
\hline$<1$ ano & 26 & 73 & 158 & 183 & 09 & 05 & 60 & 71 \\
\hline $01-04$ anos & 139 & 441 & 283 & 294 & 02 & 07 & 154 & 336 \\
\hline $05-09$ anos & 312 & 845 & 229 & 230 & 01 & 06 & 274 & 603 \\
\hline $10-14$ anos & 183 & 1.371 & 135 & 301 & 01 & 30 & 182 & 786 \\
\hline $15-19$ anos & 45 & 407 & 137 & 338 & 11 & 26 & 86 & 282 \\
\hline Total & 705 & 3.137 & 942 & 1.346 & 24 & 74 & 756 & 2.078 \\
\hline
\end{tabular}

Fonte: DATASUS [acesso 2015 Jan 24]. Disponível em: http://dtr2004.saude.gov.br/sinanweb/tabnet/dh?sinannet/violencia/bases/violebrnet.def

Quadro 1. População e serviços de atendimento às crianças e adolescentes em situação de violência sexual mencionados pelos gestores - Fortaleza, Belém, Campo Grande, Porto Alegre, 2012.

\begin{tabular}{|c|c|c|c|}
\hline $\begin{array}{c}\text { Capital e população de } \\
\text { crianças e adolescentes } \\
\text { (0 a } 19 \text { anos) }\end{array}$ & $\begin{array}{l}\text { Unidades de referência } \\
\text { (gestão municipal) }\end{array}$ & $\begin{array}{l}\text { Unidades hospitalares de } \\
\text { referência (gestão estadual/ } \\
\text { federal) }\end{array}$ & $\begin{array}{c}\text { Unidades de atendimento } \\
\text { psicossocial para crianças e } \\
\text { adolescentes (gestão municipal) }\end{array}$ \\
\hline $\begin{array}{l}\text { Fortaleza } \\
778.534\end{array}$ & $\begin{array}{l}\mathrm{N}=2 \\
\text { - Hospital da Mulher de } \\
\text { Fortaleza; } \\
\text { - Hospital Distrital Gonzaga } \\
\text { Mota de Messejana } \\
\text { (HDGMM). }\end{array}$ & $\begin{array}{l}\mathrm{N}=1 \\
\text { - Hospital Infantil Albert Sabin } \\
\text { (HIAS). }\end{array}$ & $\begin{array}{l}N=2 \\
\cdot 2 \text { CAPSi. }\end{array}$ \\
\hline $\begin{array}{l}\text { Belém } \\
450.974\end{array}$ & $\begin{array}{l}\mathrm{N}=1 \\
\text { - Casa Dia Centro de Ats } \\
\text { em Doenças Infecciosas } \\
\text { Adquiridas. }\end{array}$ & $\begin{array}{l}\mathrm{N}=1 \\
\text { - Programa PROPAZ da Santa } \\
\text { Casa de Misericórdia do Pará } \\
(\text { FSCMP). }\end{array}$ & $\begin{array}{l}\text { N=1 } \\
\cdot 1 \text { CAPSi. }\end{array}$ \\
\hline $\begin{array}{l}\text { Campo Grande } \\
248.310\end{array}$ & $\begin{array}{l}\text { N=10 } \\
\text { - } 6 \text { Centros Regionais de } \\
\text { Saúde (CRS) de Pronto } \\
\text { Atendimento ( } 24 \text { horas); } \\
\text { - } 3 \text { Unidades de Pronto } \\
\text { Atendimento (UPA); } \\
\text { - } 1 \text { Centro de Doenças } \\
\text { Infectoparasitárias (CEDIP) no } \\
\text { Hospital Dia. }\end{array}$ & $\begin{array}{l}\mathrm{N}=3 \\
\text { - Hospital Universitário Maria } \\
\text { Aparecida Pedrossian (NHU/ } \\
\text { UFMS; } \\
\text { - Santa Casa de Misericórdia; } \\
\text { - Hospital Regional de Mato } \\
\text { Grosso do Sul. }\end{array}$ & $\begin{array}{l}\mathrm{N}=2 \\
-1 \text { CAPSi; } \\
\text { - } 1 \text { CAPS Pós-Trauma. }\end{array}$ \\
\hline $\begin{array}{l}\text { Porto Alegre } \\
367.306\end{array}$ & $\begin{array}{l}\mathrm{N}=2 \\
\text { - Centro de Referência de } \\
\text { Atendimento Infantojuvenil } \\
\text { do Hospital Materno-Infantil } \\
\text { Presidente Vargas (HMIPV); } \\
\text { - Serviço de Proteção à } \\
\text { Criança (SPC). }\end{array}$ & $\begin{array}{l}\text { N=3 } \\
\text { - Hospital de Clínicas de Porto } \\
\text { Alegre; } \\
\text { - Hospital Fêmina; } \\
\text { - Hospital Nossa Senhora da } \\
\text { Conceição. }\end{array}$ & $\begin{array}{l}\mathrm{N}=2 \\
\cdot 2 \text { CAPSi. }\end{array}$ \\
\hline
\end{tabular}

Fonte de dados da população: Censo IBGE 2010. 
Na rede pública municipal de saúde de Porto Alegre, destacou-se o Centro de Referência de Atendimento Infantojuvenil (CRAI), do Hospital Materno-Infantil Presidente Vargas (HMIPV), que, além dos cuidados de saúde, oferecia atendimento integrado ao IML, para perícia médica, psiquiátrica e psicológica, e um posto da delegacia especializada no atendimento a crianças e adolescentes, apresentando uma média de 1,5 mil atendimentos por ano. O único serviço de acompanhamento ambulatorial (Serviço de Proteção à Criança), sediado no Centro de Saúde Instituto de Assistência e Proteção à Infância (IAPI), contava com pequena equipe multiprofissional.

Entretanto, mesmo entre os serviços de referência citados, nem todos estavam em pleno funcionamento. Segundo os gestores, em Fortaleza, a referência para atendimento com maior complexidade concentrava-se no Hospital da Mulher de Fortaleza. Todavia, à época da coleta de dados, esse serviço recém-inaugurado ainda não iniciara o atendimento, sendo este realizado no Hospital Distrital Gonzaga Mota de Messejana (HDGMM) e no Hospital Infantil Albert Sabin, da rede estadual.

Sobre o atendimento de saúde mental, a despeito da considerável população de crianças e adolescentes e de casos de VS, em Belém, havia apenas um Centro de Atenção Psicossocial Infantil (CAPSi), e este, segundo a gestão central, não realizava trabalho voltado para crianças e adolescentes em situação de violência. Em Campo Grande, foi referido que, além do CAPSi, havia um Centro de Atenção Psicossocial (CAPS) Pós-Trauma, que era um dos únicos do País especializado no atendimento de transtornos e traumas decorrentes da vivência de violências.

\section{Inserção do setor Saúde em fluxo intersetorial de atendimento}

Fortaleza apresentou um fluxo intersetorial para atendimento às situações de violência publicado no Protocolo de Atendimento ao Adolescente com Avaliação de Risco e Vulnerabilidade, da Secretaria Municipal de Saúde (SMS). O fluxo para atendimento inclui a VS e prevê portas de entrada na rede (serviços de saúde, delegacias, escola e Conselho Tutelar), além de fluxo específico para atendimento de adolescentes. O documento apresenta a rede de apoio e proteção discriminada em: assistências médica, psicossocial, social, jurídica e policial, além de perícia médica, conselhos, fóruns/redes e órgãos de defesa, e centros educacionais. O mesmo fluxo também se encontra inserido nas linhas de cuidado na atenção à saúde da criança, da SMS de Fortaleza ${ }^{21}$.

Os fluxos e encaminhamentos que envolvem as unidades de saúde, assim como os realizados pelas instituições do Sistema de Garantia de Direitos eram articulados pelo Centro de Referência de Direitos Humanos de Crianças e Adolescentes, que monitora as denúncias do Disque 100 e dos Conselhos Tutelares de Fortaleza. Destaca-se a articulação das unidades de saúde, assim como: a Delegacia Especializada de Combate à Exploração de Crianças e Adolescentes, o Centro de Referência Especializado de Assistência Social, os Conselhos Tutelares, a Defensoria Pública e o projeto não governamental Vira Vida, além da Rede Aquarela, da Secretaria Municipal de Direitos Humanos.

Em Belém, depoimentos de representantes das secretarias municipais de educação, de saúde e da Fundação Papa João XXIII asseguraram a inexistência de fluxo intersetorial pactuado e vigente. Contudo, um representante da Saúde informou que, anteriormente, um fluxo de atendimento intitulado "Protocolo de Atenção Integral a crianças e adolescentes vítimas de violência: uma abordagem interdisciplinar na Saúde" fora elaborado sem pactuação com a secretaria municipal de saúde, sugerindo conflitos entre a articulação estadual e a municipal. Todavia, esse fluxo não chegou a ser implantado.

"A secretaria nunca foi chamada para pactuar. Foi chamada para receber o atendimento de protocolo de crianças vítimas de violência pelo Estado. [...] Fiquei surpresa com o protocolo, já estava pronto". (representante da SMS)

A secretaria de saúde, por sua vez, por meio do Núcleo de Prevenção da Violência Doméstica e Promoção da Paz, esboçou um "manual de atendimento" para construir e pactuar um fluxo, tendo realizado uma oficina com a participação de representantes da Segurança Pública (delegacias) e da 
Assistência Social. A publicação foi distribuída nas capacitações de profissionais das unidades de saúde, mas encontrou problemas de implantação devido à rotatividade dos profissionais.

Em Campo Grande, inexistia um fluxo pactuado intersetorialmente. Havia um protocolo de atendimento às crianças e aos adolescentes em situações de VS válido para as unidades de saúde.

"Qualquer unidade, dentro do setor Saúde, que atenda um caso, suspeito ou confirmado, sabe exatamente para onde encaminhar, o que tem que fazer com a família e com o paciente. Sabe que tem que comunicar ao Conselho e encaminhar esse caso para uma unidade de referência". (representante da SMS)

Em Porto Alegre, em 2012, não havia fluxo intersetorial. As ações se concentravam setorialmente em: Saúde, Segurança Pública, Direitos Humanos e Assistência Social, e não eram específicas para VS. Representantes da SMS reafirmaram a inexistência de fluxo ou protocolo que orientasse esse atendimento nas unidades de saúde.

\section{Oferta de atendimentos às crianças e adolescentes em situação de VS}

O risco de gravidez decorrente de VS varia entre 0,5\% e 5\%, e pode representar uma segunda forma de violência. Esta realidade é ainda mais delicada quando as vítimas são adolescentes. A complexidade desta situação, envolvendo questões éticas e legais, além dos danos por ela provocados, pode ser evitada, em muitos casos, com a AEH. Neste sentido, os gestores de saúde têm a responsabilidade de garantir a disponibilidade e o acesso adequado à $A E H^{14}$.

Outra medida protetora refere-se às profilaxias de DST/Aids e hepatites virais, indicada nas situações de exposição com risco de transmissão, independentemente da presença ou da gravidade das lesões físicas, e da idade ${ }^{14}$.

\section{Anticoncepção de emergência hormonal (AEH)}

Em Fortaleza, representante da SMS informou que uma das unidades de atenção especializada no atendimento de HIV e Aids - o Serviço de Atendimento Especializado (SAE) - e o HDGMM ofereciam a AEH.

Em Belém, representante da SMS citou a Santa Casa de Misericórdia e a Casa Dia como unidades de atenção especializada no atendimento às DST/Aids que faziam essa oferta.

Em Campo Grande, a representante da Saúde informou que todas as unidades atendiam a esse procedimento. Os três hospitais vinculados às outras esferas de governo também ofertavam a AEH. Também foi enfatizada a baixa utilização desse medicamento, refletindo os preconceitos e as influências religiosas dos próprios profissionais, que omitem a existência e a disponibilidade do medicamento.

"Será que é preconceito? '- Não vou dar, não vou nem falar que tem. Eu não quero trabalho, eu não quero esse pessoal aqui, me pedindo isso, porque eu vou ter que inserir num acompanhamento, no planejamento familiar'. Ainda temos que ver o que é. Porque é muito pouco uso da AEH. É um método e deveria estar sendo utilizado". (representante da SMS)

A entrevistada citou, ainda, a articulação política da ala religiosa conservadora para que o medicamento não fosse distribuído.

"Semana passada teve uma reunião de alguns candidatos a vereadores com o arcebispo aqui da igreja [...] e fizeram propostas para o arcebispo, para a igreja católica. E uma delas é a proibição da distribuição da AEH". (representante da SMS)

Em Porto Alegre, a SMS informou que o CRAI do HMIPV e a unidade básica onde se insere o IAPI ofertavam a $\mathrm{AEH}$, além de todas as unidades básicas e centros de saúde. 


\section{Interrupção legal da gravidez (ILG)}

Em Fortaleza, de acordo com os depoimentos dos profissionais de saúde, os hospitais HDGMM e Nossa Senhora da Conceição realizavam essa intervenção, e o Hospital da Mulher de Fortaleza também realizaria, futuramente, esse procedimento. Todavia, as informações indicam que, mesmo nessas unidades, havia muita descontinuidade da oferta e resistência das equipes.

"Não são todos os profissionais que aceitam. Existem, nas equipes, alguns médicos que fazem e outros não. Começam a fazer um trabalho de organização com os profissionais que já estão fazendo. Mas alguns profissionais, por motivo religioso, ainda não concordam". (profissional da SMS)

Em Belém, somente o Hospital da Santa Casa de Misericórdia (estadual) era referência para realizar a ILG, configurando completa ausência de oferta na rede municipal.

Representante da SMS de Campo Grande informou que somente o Hospital Regional de Mato Grosso do Sul realizava tal atendimento. Nenhuma unidade municipal assumiu essa ação, nem mesmo o Hospital da Mulher.

Representante da SMS de Porto Alegre informou que as mesmas unidades hospitalares que constituíam referência para o atendimento às crianças e aos adolescentes em situação de VS (HMIPV, Hospital de Clínicas de Porto Alegre, Fêmina e Nossa Senhora da Conceição) realizavam a ILG, no entanto, apenas a primeira estava sob gestão municipal.

\section{Oferta e fluxo para profilaxias de DST/Aids e hepatites virais}

Em Fortaleza, foram citados apenas dois serviços municipais que prestavam esse atendimento: 0 HDGMM e o SAE. O hospital realizava o acompanhamento ambulatorial e, para imunoprofilaxia das hepatites virais, apenas um dos 11 SAE do município (Centro de Saúde da Família Carlos Ribeiro), além do Centro de Referência de Imunobiológicos Especiais (CRIE), de gestão estadual, prestavam esse atendimento.

Em Belém, foram apontadas duas unidades que realizavam o atendimento: no CRIE, que funciona no Hospital da Santa Casa de Misericórdia; e a Casa Dia (unidade municipal especializada em atendimento das DST/Aids), que ofertava imunoprofilaxia para as hepatites virais.

Em Campo Grande, foram citadas como referência as mesmas unidades para o atendimento às crianças e aos adolescentes em situação de VS, acrescidas do Hospital Dia/Centro Especializado de Doenças Infectoparasitárias (CEDIP). Haveria, portanto, dez unidades municipais ofertando esse serviço. Além delas, os três hospitais mencionados também realizavam essa profilaxia. As dez unidades mencionadas anteriormente disponibilizariam a imunoglobulina. O CRIE, instalado no Hospital Regional de Mato Grosso do Sul, também ofertava todas as vacinas e realizava o protocolo completo.

Em Porto Alegre, representante da SMS citou como referência as mesmas unidades hospitalares que realizavam a AEH e a ILG, frisando que somente uma era municipal, o HMIPV. Os entrevistados informaram que todas as unidades de referência encaminhavam os casos para o CRIE de referência regional.

\section{Discussão}

Embora as quatro capitais possuam uma rede de saúde complexa e com realidades distintas, o quantitativo de unidades de saúde que atendem às demandas clínicas e psicossociais das situações de VS de crianças e adolescentes é bastante insuficiente, se for considerada a magnitude desse fenômeno e as exigências legais para esse atendimento ${ }^{22}$. Paulatinamente, vem ocorrendo nacionalmente um aumento do número de serviços voltados para a atenção às mulheres em situação de VS, que acabam por incluir crianças e adolescentes ${ }^{23}$; todavia, observam-se retrocessos, pois serviços especializados 
com expertise para atendimento ao segmento infantojuvenil têm sido precarizados ou fechados, e os de atendimento a mulheres ainda apresentam baixa cobertura para o território nacional ${ }^{24}$.

Nesse contexto, observa-se que a organização dessa atenção é desigual entre as regiões brasileiras. A afirmação dos gestores de que todas as unidades da rede básica estariam aptas a identificar, notificar e encaminhar os casos para unidades de maior complexidade demandaria uma investigação mais apurada quanto à sua fidedignidade. Tal afirmação pode significar cenários distintos: um de real investimento na capacitação dos profissionais para atuar, e outro em que o caso (quando identificado) é "atendido" para ser simplesmente "enviado" a outro serviço.

Em três dos quatro municípios, o atendimento de referência ocorria por meio de serviços de emergência situados na atenção terciária. Em contrapartida, permanece nessas, e na maioria das capitais, a necessidade de capilarização do atendimento da atenção primária em saúde ${ }^{25}$.

As experiências de centros integrados de atendimento - como as do CRAI de Porto Alegre e do Programa PROPAZ, em Belém (estadual) - capazes de reunir, em um mesmo espaço físico, o atendimento médico, o psicossocial, o de perícia legal e de denúncia policial destacam-se, positivamente, pela integralidade do atendimento e pela intersetorialidade da atenção.

A participação das instâncias estaduais e federais observada pode representar uma articulação em rede das unidades de saúde nessa atenção, assim como também significar uma relativa ausência da gestão municipal, responsável pela execução da política voltada a esse segmento, como verificado em Belém.

A oferta de serviços de saúde mental para a atenção às crianças e aos adolescentes em situação de VS em todas as quatro capitais, para além do cumprimento ao previsto nas políticas referentes ${ }^{1}$, qualifica a atenção prestada. Todavia, como têm denunciado vários estudos em todo o País, a oferta ainda é baixa, com poucos CAPSi para populações de dimensões metropolitanas ${ }^{26}$. Como problematiza estudo recente ${ }^{27}$, a existência de CAPSi não garante a oferta de psicoterapia para as crianças e os adolescentes que sofreram VS. Segundo diretriz governamental, esses serviços devem atender a transtornos mentais severos e/ou persistentes, mas tais critérios não são claramente definidos e tal julgamento é feito pelo profissional. Assim, os casos que não são reconhecidos nessa condição não encontram serviço de referência na rede pública de saúde para atendimento psicoterápico. Nesse cenário restritivo, destaca-se a experiência pioneira de Campo Grande, com um CAPS especializado no atendimento aos traumas e transtornos decorrentes das violências.

Sobre a inserção do setor Saúde em um fluxo intersetorial, observa-se o esforço dos municípios quanto ao estabelecimento de vínculos formalizados com os demais setores para compor uma rede integrada de atenção a vítimas de violência. Chama a atenção que três municípios possuíam protocolos publicados e divulgados ou em fase de elaboração, mas cujo processo de implementação enfrentava problemas. Como a literatura aponta, a baixa articulação intersetorial favorece a segmentação da atenção, fragiliza a integralidade do cuidado e expõe esse grupo a novas modalidades de violência, destacando-se a institucional ${ }^{28}$. Em contrapartida, observa-se o aumento de até quatro vezes no número de atendimentos, quando existe um protocolo integrado com atuação intersetorial, evidenciando que a existência da oferta influi na busca por atendimento ${ }^{29}$. A elaboração de fluxos operacionais torna-se relevante para qualificar o processo de trabalho de profissionais que se articulam em rede e contribuir para responder às exigências legais, além de reorientar práticas ${ }^{30}$.

As dificuldades encontradas nessa pesquisa, em relação à $A E H$, também são mencionadas na literatura ${ }^{31,32}$. Nota-se a ausência de capilaridade da oferta nas unidades básicas de saúde, centralizada nas unidades de referência. A insuficiência e a demora da AEH conduzem à baixa eficácia, podendo contribuir para o aumento do número de gestações resultantes de VS. Como foi verificado em pesquisas, mesmo nos municípios em que a medicação encontra-se nas unidades, a oferta é comprometida por mitos, preconceitos, convicções éticas e religiosas dos componentes das equipes de saúde, bem como sofre pressão de movimentos religiosos conservadores ${ }^{33}$. Estudos recentes revelam que a oferta da AEH em situação de VS ainda tem cobertura insuficiente, mesmo nos Estados Unidos. Patel et al. ${ }^{34}$ analisam, em amostra representativa dos hospitais americanos, a oferta da AEH, entre os períodos de 2004 e 2009, e revelam que a provisão do medicamento nos casos de VS cresceu apenas $1 \%$, chegando a $64 \%$. 
Sobre a ILG, o que foi observado também é enfatizado em pesquisas sobre o tema que denunciam as flutuações da oferta do atendimento pela baixa adesão dos profissionais de saúde, que alegam "objeção de consciência", evidenciando o confronto entre tal prerrogativa profissional e os direitos sexuais e reprodutivos das mulheres ${ }^{35}$. Embora no Brasil o Ministério da Saúde tenha normatizado os procedimentos para a interrupção de gravidez por VS ${ }^{14,36}$, como em outros estudos ${ }^{37}$, observa-se, também, a interferência religiosa para coibir esse atendimento.

Todavia, é mister lembrar a responsabilidade da gestão em garantir, nesses serviços, os atendimentos clínico, ginecológico, cirúrgico e psicossocial, contando com: serviço de apoio laboratorial; apoio diagnóstico e de farmácia hospitalar; e notificação compulsória institucionalizada ${ }^{38}$.

Do mesmo modo, a oferta das profilaxias de DST/Aids e hepatites virais se mostra insuficiente, muitas vezes sem definição de fluxo e vinculada a serviços de referência. Nos EUA, há oferta de profilaxia para HIV em $65 \%$ dos hospitais de emergência americanos, e em $77 \%$ para outras DSTs ${ }^{39}$. Lá, o problema encontrado é a baixa adesão aos protocolos de acompanhamento ambulatorial ${ }^{40}$. Em nosso estudo, em três das quatro capitais, o atendimento era concentrado em poucos serviços. Apesar das severas consequências físicas e emocionais das DSTs decorrentes da VS, o despreparo dos provedores e dos profissionais de saúde, no País, é apontado como um dos obstáculos à precocidade da intervenção ${ }^{41}$.

Finalmente, ressalva-se que o estudo apresenta limitações por ancorar-se na declaração dos gestores (ainda que confrontadas por documentação oficial), não tendo sido realizadas visitas aos serviços ou checadas as ações citadas a fim de verificar sua veracidade. Além disso, não inclui as opiniões dos familiares sobre o atendimento.

\section{Considerações finais}

A atenção à saúde de crianças e adolescentes em situação de VS é regulada por normativas ainda não satisfatoriamente efetivadas pela gestão por meio de práticas profissionais capazes de minimizar as severas repercussões que essa violência acarreta à saúde desse grupo, devendo se articular mediante oferta organizada e qualificada, que assegure o exercício pleno dos direitos humanos ${ }^{14}$. Nota-se a necessidade de fortalecimento do diálogo intersetorial para consolidação e revisão de fluxos e protocolos, evitando fragmentação da atenção, desperdício de tempo e mais sofrimento às famílias. As ofertas de psicoterapia, AEH, ILG e das profilaxias de DST/Aids e hepatites virais devem assegurar direitos conquistados e garantidos por lei, na perspectiva da proteção integral de crianças e adolescentes, o que envolve um processo contínuo de qualificação e supervisão aos profissionais e gestores de saúde.

\section{Colaboradores}

Os autores participaram, igualmente, de todas as etapas de elaboração do artigo. 


\section{Referências}

1. Ministério da Saúde (BR). Secretaria de Políticas de Saúde. Violência intrafamiliar: orientações para prática em serviço. Brasília (DF): MS; 2002.

2. Organização Mundial de Saúde. Relatório mundial sobre violência e saúde. Genebra: OMS; 2002.

3. Mello LCA, Dutra E. Abuso sexual contra crianças: em busca de uma compreensão centrada na pessoa. Rev Abordagem Gestalt. 2008; 14(1):39-47.

4. Avanci J, Assis S, Oliveira R, Pires T. Quando a convivência com a violência aproxima a criança do comportamento depressivo. Cienc Saude Colet. 2009; 14(2): 383-94.

5. Ministério da Saúde (BR). Secretaria de Atenção à Saúde, Departamento de Ações Programáticas Estratégicas. Direitos sexuais e direitos reprodutivos: uma prioridade do governo. Brasília (DF): MS; 2005.

6. Mattar LD. Reconhecimento jurídico dos direitos sexuais: uma análise comparativa com os direitos reprodutivos. Sur Rev Int Direitos Human. 2008; 5(8):60-83.

7. Cordeiro F, Heilborn ML, Cabral CS, Moraes CL. Entre negociação e conflito: gênero e coerção sexual em três capitais brasileiras. Cienc Saude Colet. 2009; 14(4):1051-62.

8. Fundo das Nações Unidas para a Infância. Uma análise estatística sobre violência contra crianças [Internet]. Portugal; 2014 [acesso 2015 Mar 12]. Disponível em: http://www. unicef.pt/violencia-criancas/violencia-criancas-2014.html

9. Secretaria de Direitos Humanos. Presidência da República (BR). Disque 100 - Disque Direitos Humanos/módulo criança e adolescente. Brasília (DF): SDH-PR; 2014.

10. Minayo MCS. A inclusão da violência na agenda da saúde: trajetória histórica. Cienc Saude Colet. 2006; 11 Supl:1259-67.

11. Ministério da Saúde (BR). Sistema de Informação de Agravo de Notificação. [Internet] [acesso 2013 Jul 17]. Disponível em: http://dtr2004.saude.gov.br/sinanweb/tabnet/ dh?sinannet/violencia/bases/violebrnet.def

12. Ministério da Saúde (BR). Secretaria de Atenção à Saúde, Departamento de Ações Programáticas e Estratégicas. Linha de cuidado para a atenção integral à saúde de crianças, adolescentes e suas famílias em situação de violência. Brasília (DF): MS; 2010.

13. Ministério da Justiça (BR). Estatuto da criança e do adolescente. Lei no 8.069, de 13 de julho 1990. Dispõe sobre o Estatuto da criança e do adolescente e dá outras providências. Brasília (DF): MJ; 1990.

14. Ministério da Saúde (BR). Secretaria de Atenção à Saúde. Prevenção e tratamento dos agravos resultantes da violência sexual contra mulheres e adolescentes: norma técnica. $3 a$ ed. Brasília (DF): MS; 2012.

15. Deslandes SF, Mendes $\mathrm{CH}$, Pires TO, Campos DS. Use of the nominal group technique and the delphi method to draw up evaluation indicators for strategies to deal with violence against children and adolescents in Brazil. Rev Bras Saude Mater Infant. 2010; 10 Supl:29-37.

16. Deslandes SF, Mendes CHF, Luz ES. Análise de desempenho de sistema de indicadores para o enfrentamento da violência intrafamiliar e exploração sexual de crianças e adolescentes. Cienc Saude Colet. 2014; 19(3):865-74.

17. Yin RK. Estudo de caso: planejamento e métodos. Porto Alegre: Bookmann; 2010.

18. Gil AC. Como elaborar projetos de pesquisa. 5a ed. São Paulo: Atlas; 2008.

19. Gibbs G. Análise de dados qualitativos. Porto Alegre: Artmed; 2009. 
20. Instituto de Pesquisa Econômica Aplicada. Atlas do desenvolvimento humano no Brasil. Perfil [Internet] Brasília: PNUD, Ipea, FJP [acesso 2015 Out 20]. Disponível em: www. atlasbrasil.org.br

21. Secretaria Municipal de Saúde de Fortaleza. Linhas de cuidado na Atenção à Saúde da Criança. Fortaleza: SMS; 2012.

22. Souza CS, Costa MCO, Assis SG, Musse JO, Sobrinho CN, Amaral MTR. Sistema de Vigilância de Violências e Acidentes/VIVA e a notificação da violência infanto-juvenil, no Sistema Único de Saúde/SUS de Feira de Santana-Bahia, Brasil. Cienc Saude Colet. 2014; 19(3):773-84.

23. Andalaft Neto J, Faúndes $A$, Osis MJD, Pádua KS. Perfil do atendimento à violência sexual no Brasil. Femina. 2012; 40(6):301-6.

24. Lima CA, Deslandes SF. Violência sexual contra mulheres no Brasil: conquistas e desafios do setor saúde na década de 2000. Saude Soc. 2014; 23(3):787-800.

25. Ministério da Saúde (BR). Portaria n 936, de 19 de maio de 2004. Dispõe sobre a estruturação da Rede Nacional de Prevenção da Violência e Promoção da Saúde e a implantação e implementação de Núcleos de Prevenção à Violência em estados e municípios. Brasília (DF): MS; 2004.

26. Couto MCV, Duarte CS, Delgado PGG. A saúde mental infantil na Saúde Pública brasileira: situação atual e desafios. Rev Bras Psiquiatr. 2008; 30(8):390-8.

27. Hohendorff JV, Habigzang LF, Koller SH. Psicoterapia para crianças e adolescentes vítimas de violência sexual no sistema público: panorama e alternativas de atendimento. Psicol Cienc Prof. 2015; 35(1):182-98.

28. Pelisoli C, Pires JPM, Almeida ME, Dell'Aglio DD. Violência sexual contra crianças e adolescentes: dados de um serviço de referência. Temas Psicol. 2010; 18(1):85-97.

29. Campos MAMR, Schor N, Anjos RMP, Laurentiz JC, Santos DV, Peres F. Violência sexual: integração saúde e segurança pública no atendimento imediato à vítima. Saude Soc. 2005; 14(1):101-9.

30. Associação Brasileira de Magistrados. Proteção integral e atuação em rede na garantia dos direitos de crianças e adolescentes. São Paulo: ABPM; 2010.

31. Gold SN, Hyman SM, Andrés-Hyman RC. Family of origin environments in two clinical samples of survivors of intra-familial, extra-familial, and both types of sexual abuse. Child Abuse Neglect. 2004; 28(1):1199-212.

32. Figueiredo R, Bastos S, Telles JL. Perfil da distribuição da contracepção de emergência para adolescentes em municípios do estado de São Paulo. Rev Bras Cresc Desenv Hum. $2012 ; 22(1): 1-15$.

33. Moraes SP, Vitalle MSS. Direitos sexuais e reprodutivos na adolescência. Rev Assoc Med Bras. 2012; 58(1):48-52.

34. Patel A, Tilmon S, Bhogireddy V, Chor J, Patel D, Keith L. Emergency contraception after sexual assault: changes in provision from 2004 to 2009. J Reprod Med. 2012; 57(34):98-104.

35. Zordo S. Representações e experiências sobre aborto legal e ilegal dos ginecologistasobstetras trabalhando em dois hospitais maternidade de Salvador da Bahia. Cienc Saude Colet. 2012; 17(7):1745-54.

36. Portaria $n^{\circ} 1.508$, de $1^{\circ}$ de setembro de 2005. Dispõe sobre o procedimento de justificação e autorização da interrupção da gravidez nos casos previstos em lei, no âmbito do Sistema Único de Saúde - SUS. Diário Oficial da União. 2 Set 2005. 
37. Farias RS, Cavalcanti LF. Atuação diante das situações de aborto legal na perspectiva dos profissionais de saúde do Hospital Municipal Fernando Magalhães. Cienc Saude Colet. 2012; 17(7):1755-63.

38. Portaria $n^{\circ} 528$, de $1^{\circ}$ de abril de 2013. Define regras para habilitação e funcionamento dos Serviços de Atenção Integral às Pessoas em Situação de Violência Sexual no âmbito do Sistema Único de Saúde (SUS). Diário Oficial da União. 2 Abr 2013.

39. Patel A, Roston A, Tilmon S, Stern L, Roston A, Patel D, Keith L. Assessing the extent of provision of comprehensive medical care management for female sexual assault patients in US hospital emergency departments. Int J Gynaecol Obstet. 2013; 123(1):24-8.

40. Krause KH, Lewis-O'Connor A, Berger A, Votto T, Yawetz S, Pallin DJ, Baden LR. Current practice of HIV postexposure prophylaxis treatment for sexual assault patients in an emergency department. Women Health Issues. 2014; 24(4):e407-12.

41. Drezett J. Violência sexual como problema de saúde pública. In: Taquette SR, organizadora. Violência contra a mulher adolescente-jovem. Rio de Janeiro: Ed. UERJ; 2007. p. 81-90.

Deslandes SF, Vieira LJES, Cavalcanti LF, Silva RM. Cuidado de la salud para niños y adolescentes víctimas de violencia sexual en cuatro capitales brasileñas. Interface (Botucatu). 2016; 20(59):865-77.

El artículo analiza el cuidado de la salud de los niños y adolescentes víctimas de violencia en servicios de salud pública municipal en cuatro ciudades - Porto Alegre (Rio Grande do Sul), Belém (Pará), Fortaleza (Ceará) y Campo Grande (Mato Grosso do Sul) - , centrándose en la disponibilidad de servicios y la prestación de consultas de anticoncepción hormonal de emergencia $(\mathrm{AHE})$, profilaxis de enfermedades de transmisión sexual e interrupción del embarazo. El estudio del caso se realizó a través de entrevistas semi-estructuradas con veinte representantes de los departamentos de salud locales y análisis de documentos. Aquí, fue posible observar que la prestación de atención entre las capitales es desigual. Aunque la demanda es alta, existe poca oferta de servicios en tres de las ciudades analizadas. Por otra parte, la atención especializada, como AHE y el aborto legal todavía se enfrentan a la resistencia de los profesionales y sectores religiosos. Se destacan experiencias innovadoras de dos Centros Integrados de Atención Psicosocial y un Centro de Atención Pos-Trauma.

Palabras clave: Cuidado de la Salud. Niño y adolescente. Violencia sexual. Servicios de Salud. 
